Supplement of Solid Earth, 11, 2463-2485, 2020

https://doi.org/10.5194/se-11-2463-2020-supplement

(c) Author(s) 2020. This work is distributed under

the Creative Commons Attribution 4.0 License.

(c) (i)

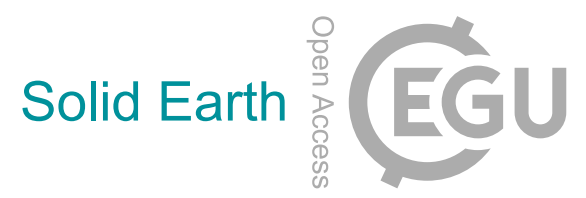

Supplement of

\title{
Birth and closure of the Kallipetra Basin: Late Cretaceous reworking of the Jurassic Pelagonian-Axios/Vardar contact (northern Greece)
}

Lydia R. Bailey et al.

Correspondence to: Lydia R. Bailey (lydiabailey@email.arizona.edu)

The copyright of individual parts of the supplement might differ from the CC BY 4.0 License. 


\section{Supplementary data}
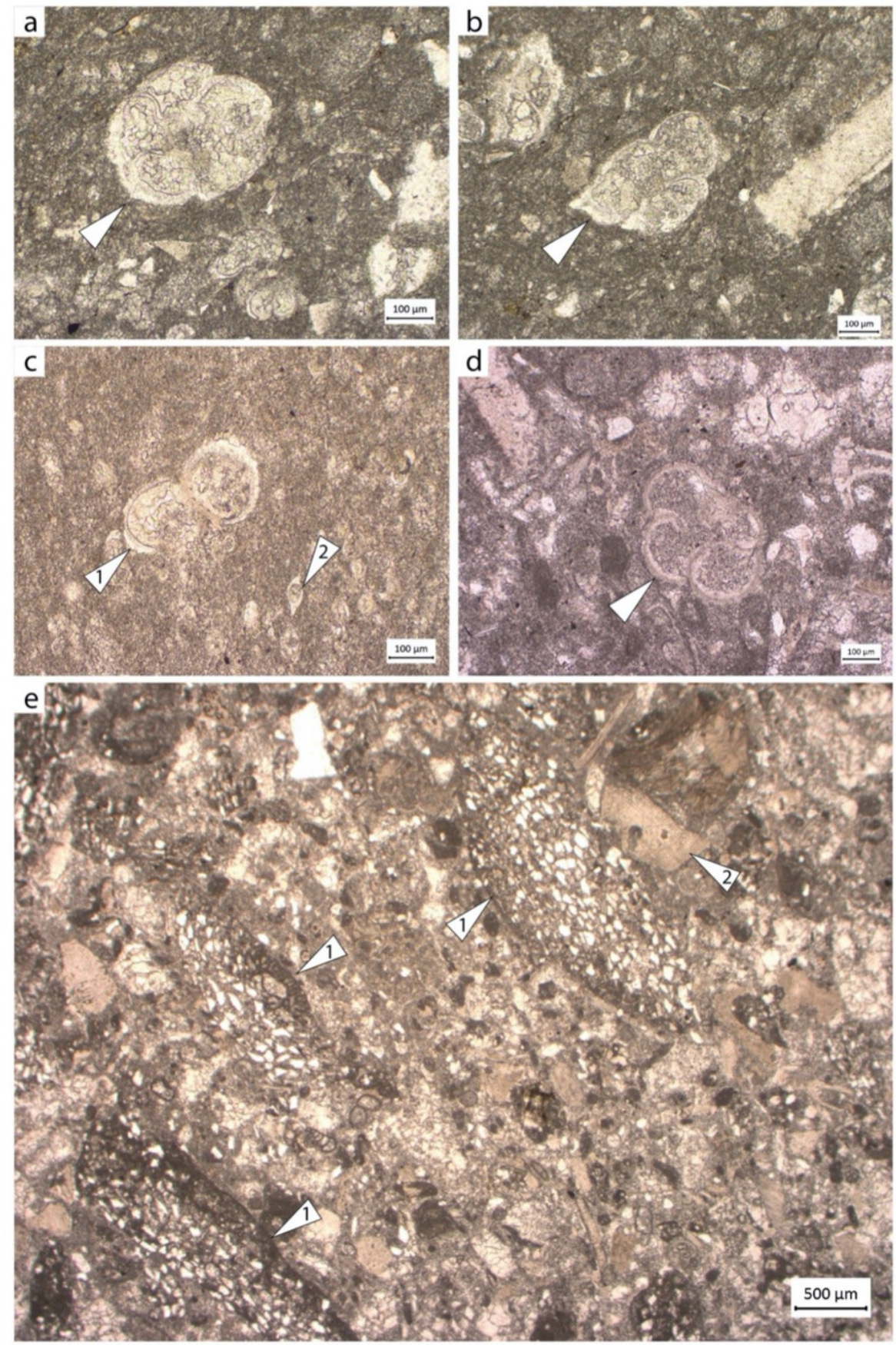

Figure S1: (a) Sample M2-TS1 Helvetoglobotruncana helvetica; (b) Sample M2-TS1 Dicarinella sp.; (c) Sample M2-TS2, $1=$ Whiteinella, 2= radiolarian; (d) Sample M2-TS3 Whiteinella; (e) M2-TS3, 1 = Orbitolinid, 2 = Echinoderm. 


\section{Asomata M2 Section}

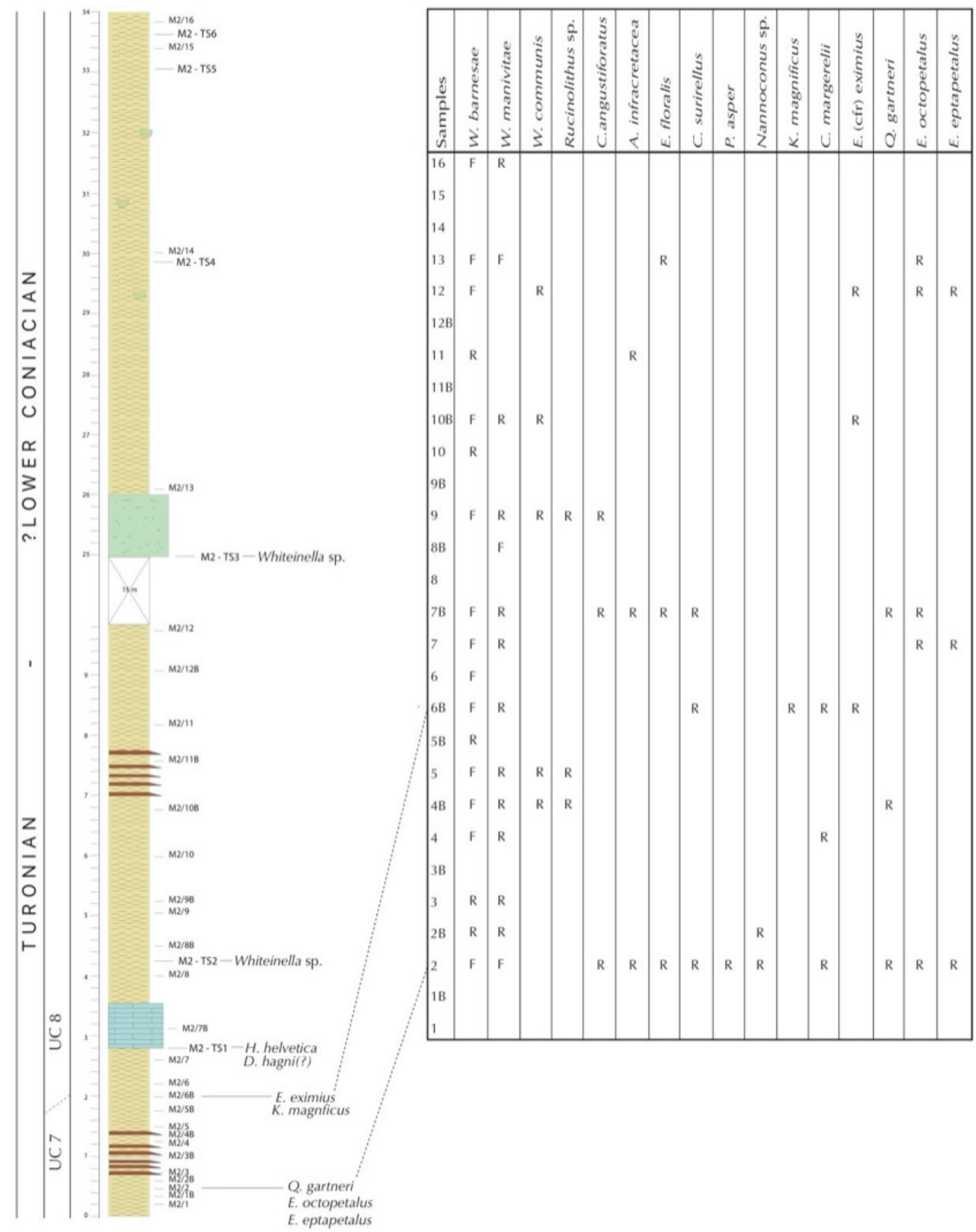

Figure S2: Biostratigraphic section M2 showing sample depths, observed foraminifera, and observed nannoplankton. Section taken from $\mathrm{N}^{\circ}{ }^{\circ} 28$ ' 53 ' $\mathrm{E022}{ }^{\circ} 13$ ' 46". 
Supplementary Data

Table S3

\begin{tabular}{|c|c|c|c|c|}
\hline Plane & Latitude & Longitude & Dip Angle & Dip Azimuth \\
\hline Fault & 40.465041 & 22.242527 & 86 & 13 \\
\hline Fault & 40.465196 & 22.242587 & 21 & 66 \\
\hline Fault & 40.420692 & 22.193283 & 55 & 82 \\
\hline Fault & 40.425450 & 22.190061 & 34 & 354 \\
\hline Fault & 40.425248 & 22.180059 & 34 & 21 \\
\hline Fault & 40.481256 & 22.227301 & 82 & 50 \\
\hline Fault & 40.483579 & 22.230109 & 15 & 90 \\
\hline Fault & 40.483597 & 22.230115 & 18 & 23 \\
\hline Fault & 40.461326 & 22.258370 & 38 & 179 \\
\hline Fault & 40.461368 & 22.258403 & 27 & 184 \\
\hline Fault & 40.475379 & 22.247594 & 39 & 28 \\
\hline Fault & 40.474400 & 22.246642 & 24 & 24 \\
\hline Fault & 40.474049 & 22.246296 & 26 & 42 \\
\hline Fault & 40.472414 & 22.244458 & 20 & 178 \\
\hline Fault & 40.411390 & 22.196036 & 81 & 288 \\
\hline Fault & 40.412645 & 22.195346 & 26 & 148 \\
\hline Fault & 40.467859 & 22.242980 & 56 & 162 \\
\hline Fault & 40.467867 & 22.242925 & 28 & 18 \\
\hline Fault & 40.467675 & 22.242466 & 13 & 68 \\
\hline Fault & 40.437418 & 22.175634 & 43 & 131 \\
\hline Fault & 40.436383 & 22.178010 & 68 & 359 \\
\hline Fault & 40.437819 & 22.246576 & 40 & 103 \\
\hline Fault & 40.415774 & 22.228657 & 77 & 18 \\
\hline Fault & 40.415790 & 22.228687 & 4 & 334 \\
\hline Fault & 40.468674 & 22.211876 & 89 & 308 \\
\hline Fault & 40.468853 & 22.211844 & 75 & 283 \\
\hline Fault & 40.469669 & 22.210826 & 42 & 33 \\
\hline Fault & 40.472480 & 22.212549 & 78 & 239 \\
\hline Fault & 40.473195 & 22.213055 & 46 & 210 \\
\hline Fault & 40.473217 & 22.213029 & 56 & 26 \\
\hline Fault & 40.480384 & 22.216631 & 64 & 327 \\
\hline Fault & 40.479099 & 22.213695 & 87 & 61 \\
\hline Fault & 40.478039 & 22.212978 & 69 & 235 \\
\hline Fault & 40.478011 & 22.212996 & 56 & 212 \\
\hline Fault & 40.478052 & 22.212999 & 69 & 237 \\
\hline Fault & 40.479093 & 22.213693 & 85 & 60 \\
\hline Fault & 40.408432 & 22.196112 & 42 & 256 \\
\hline Foliation & 40.464575 & 22.263631 & 19 & 172 \\
\hline Foliation & 40.456710 & 22.250205 & 40 & 328 \\
\hline Foliation & 40.456529 & 22.250096 & 27 & 314 \\
\hline Foliation & 40.442747 & 22.245089 & 34 & 200 \\
\hline Foliation & 40.426324 & 22.189264 & 21 & 306 \\
\hline Foliation & 40.461935 & 22.240930 & 37 & 61 \\
\hline Foliation & 40.461861 & 22.240914 & 38 & 2 \\
\hline Foliation & 40.462222 & 22.241018 & 40 & 339 \\
\hline Foliation & 40.462324 & 22.241086 & 27 & 22 \\
\hline Foliation & 40.458361 & 22.253522 & 48 & 165 \\
\hline
\end{tabular}


Supplementary Data

Table S3

\begin{tabular}{|c|c|c|c|c|}
\hline Foliation & 40.457803 & 22.253438 & 14 & 130 \\
\hline Foliation & 40.457029 & 22.253030 & 48 & 335 \\
\hline Foliation & 40.456957 & 22.252924 & 76 & 158 \\
\hline Foliation & 40.456852 & 22.253028 & 82 & 340 \\
\hline Foliation & 40.392756 & 22.210951 & 29 & 251 \\
\hline Foliation & 40.479316 & 22.254012 & 34 & 299 \\
\hline Foliation & 40.479117 & 22.248737 & 35 & 139 \\
\hline Foliation & 40.478465 & 22.248536 & 45 & 182 \\
\hline Foliation & 40.475361 & 22.247586 & 37 & 356 \\
\hline Foliation & 40.474411 & 22.246738 & 23 & 66 \\
\hline Foliation & 40.474361 & 22.246677 & 27 & 26 \\
\hline Foliation & 40.474358 & 22.246734 & 22 & 66 \\
\hline Foliation & 40.474098 & 22.246299 & 31 & 30 \\
\hline Foliation & 40.472750 & 22.244759 & 18 & 146 \\
\hline Foliation & 40.472467 & 22.244709 & 27 & 186 \\
\hline Foliation & 40.472338 & 22.244813 & 27 & 147 \\
\hline Foliation & 40.472367 & 22.244790 & 19 & 196 \\
\hline Foliation & 40.472330 & 22.244652 & 28 & 224 \\
\hline Foliation & 40.472031 & 22.244589 & 21 & 321 \\
\hline Foliation & 40.471425 & 22.243880 & 74 & 14 \\
\hline Foliation & 40.471280 & 22.243020 & 33 & 47 \\
\hline Foliation & 40.414277 & 22.195455 & 44 & 94 \\
\hline Foliation & 40.413048 & 22.194560 & 25 & 287 \\
\hline Foliation & 40.413571 & 22.196419 & 10 & 49 \\
\hline Foliation & 40.467676 & 22.242974 & 23 & 178 \\
\hline Foliation & 40.467697 & 22.243009 & 16 & 106 \\
\hline Foliation & 40.465721 & 22.230135 & 48 & 293 \\
\hline Foliation & 40.465634 & 22.230131 & 20 & 347 \\
\hline Foliation & 40.464670 & 22.226987 & 82 & 249 \\
\hline Foliation & 40.464796 & 22.228344 & 48 & 33 \\
\hline Foliation & 40.464756 & 22.227833 & 56 & 304 \\
\hline Foliation & 40.450244 & 22.247460 & 88 & 88 \\
\hline Foliation & 40.437446 & 22.247761 & 12 & 105 \\
\hline Foliation & 40.437483 & 22.247827 & 79 & 130 \\
\hline Foliation & 40.437457 & 22.247776 & 63 & 107 \\
\hline Foliation & 40.437831 & 22.246569 & 24 & 58 \\
\hline Foliation & 40.437822 & 22.246689 & 11 & 159 \\
\hline Foliation & 40.437705 & 22.246749 & 22 & 10 \\
\hline Foliation & 40.432618 & 22.239742 & 34 & 35 \\
\hline Foliation & 40.432386 & 22.240025 & 24 & 236 \\
\hline Foliation & 40.426805 & 22.240821 & 10 & 73 \\
\hline Foliation & 40.426298 & 22.241195 & 17 & 354 \\
\hline Foliation & 40.443008 & 22.259099 & 22 & 335 \\
\hline Foliation & 40.443247 & 22.259197 & 28 & 49 \\
\hline Foliation & 40.445916 & 22.260071 & 14 & 32 \\
\hline Foliation & 40.445718 & 22.260113 & 34 & 140 \\
\hline Foliation & 40.445748 & 22.260061 & 23 & 26 \\
\hline Foliation & 40.445747 & 22.260064 & 20 & 20 \\
\hline
\end{tabular}


Supplementary Data

Table S3

\begin{tabular}{|c|c|c|c|c|}
\hline Foliation & 40.444831 & 22.260781 & 57 & 128 \\
\hline Foliation & 40.444827 & 22.260805 & 24 & 147 \\
\hline Foliation & 40.418742 & 22.244351 & 43 & 311 \\
\hline Foliation & 40.418929 & 22.244168 & 71 & 330 \\
\hline Foliation & 40.418927 & 22.244170 & 33 & 342 \\
\hline Foliation & 40.418901 & 22.244174 & 53 & 320 \\
\hline Foliation & 40.418906 & 22.244209 & 54 & 296 \\
\hline Foliation & 40.419057 & 22.241613 & 81 & 333 \\
\hline Foliation & 40.419082 & 22.241636 & 55 & 51 \\
\hline Foliation & 40.416714 & 22.232965 & 21 & 302 \\
\hline Foliation & 40.416734 & 22.232971 & 36 & 306 \\
\hline Foliation & 40.417413 & 22.234782 & 18 & 23 \\
\hline Foliation & 40.417588 & 22.235286 & 71 & 128 \\
\hline Foliation & 40.418183 & 22.237368 & 66 & 85 \\
\hline Foliation & 40.480196 & 22.220905 & 67 & 318 \\
\hline Foliation & 40.480114 & 22.220658 & 79 & 169 \\
\hline Foliation & 40.479814 & 22.219476 & 43 & 301 \\
\hline Cleavage & 40.444433 & 22.261622 & 53 & 351 \\
\hline Cleavage & 40.416311 & 22.246411 & 61 & 150 \\
\hline Cleavage & 40.416742 & 22.227183 & 89 & 303 \\
\hline Cleavage & 40.416739 & 22.227180 & 84 & 300 \\
\hline Cleavage & 40.416729 & 22.227169 & 79 & 308 \\
\hline Shear Plane & 40.480925 & 22.225507 & 41 & 65 \\
\hline Shear Plane & 40.480947 & 22.225552 & 36 & 76 \\
\hline Shear Plane & 40.464659 & 22.227007 & 51 & 81 \\
\hline Shear Plane & 40.464804 & 22.228344 & 54 & 242 \\
\hline Shear Plane & 40.441827 & 22.244630 & 22 & 331 \\
\hline Shear Plane & 40.437819 & 22.246697 & 24 & 53 \\
\hline Shear Plane & 40.432411 & 22.239894 & 24 & 324 \\
\hline Shear Plane & 40.433135 & 22.242140 & 47 & 349 \\
\hline Shear Plane & 40.433135 & 22.242167 & 28 & 11 \\
\hline Shear Plane & 40.433075 & 22.242114 & 32 & 298 \\
\hline Shear Plane & 40.443814 & 22.262142 & 41 & 244 \\
\hline Shear Plane & 40.480644 & 22.219537 & 75 & 317 \\
\hline Shear Plane & 40.480649 & 22.219570 & 54 & 222 \\
\hline Shear Plane & 40.480661 & 22.219575 & 40 & 183 \\
\hline Shear Plane & 40.479771 & 22.219035 & 58 & 186 \\
\hline Shear Plane & 40.478975 & 22.213497 & 65 & 61 \\
\hline Shear Plane & 40.479007 & 22.213430 & 73 & 182 \\
\hline Shear Plane & 40.478914 & 22.213468 & 26 & 61 \\
\hline Shear Plane & 40.478954 & 22.213444 & 57 & 20 \\
\hline Shear Plane & 40.478424 & 22.213339 & 18 & 36 \\
\hline Shear Plane & 40.478389 & 22.213321 & 21 & 11 \\
\hline Shear Plane & 40.478949 & 22.213473 & 55 & 47 \\
\hline Shear Plane & 40.478934 & 22.213488 & 42 & 349 \\
\hline Shear Plane & 40.478883 & 22.213510 & 26 & 324 \\
\hline Shear Plane & 40.476073 & 22.216853 & 26 & 305 \\
\hline Shear Plane & 40.476155 & 22.217035 & 39 & 274 \\
\hline
\end{tabular}


Supplementary Data

Table S3

\begin{tabular}{|c|c|c|c|c|}
\hline Shear Plane & 40.476186 & 22.216982 & 79 & 339 \\
\hline Shear Plane & 40.476186 & 22.216982 & 55 & 154 \\
\hline Shear Plane & 40.477391 & 22.216691 & 31 & 283 \\
\hline Shear Plane & 40.477391 & 22.216691 & 23 & 2 \\
\hline Shear Plane & 40.477391 & 22.216691 & 83 & 311 \\
\hline Shear Plane & 40.411102 & 22.197115 & 18 & 188 \\
\hline Line & Latitude & Longitude & Plunge Dip & Plunge Azimuth \\
\hline Fold Axis & 40.437943 & 22.175514 & 22 & 305 \\
\hline Fold Axis & 40.437928 & 22.174911 & 31 & 128 \\
\hline Fold Axis & 40.437316 & 22.175734 & 18 & 222 \\
\hline Fold Axis & 40.429903 & 22.189150 & 6 & 298 \\
\hline Fold Axis & 40.429779 & 22.188602 & 7 & 114 \\
\hline Fold Axis & 40.429789 & 22.188658 & 4 & 289 \\
\hline Fold Axis & 40.423700 & 22.240841 & 6 & 39 \\
\hline Fold Axis & 40.415913 & 22.246595 & 10 & 41 \\
\hline Fold Axis & 40.416250 & 22.246465 & 26 & 9 \\
\hline Fold Axis & 40.417263 & 22.245961 & 16 & 31 \\
\hline Fold Axis & 40.416645 & 22.227238 & 3 & 136 \\
\hline Fold Axis & 40.415989 & 22.228490 & 9 & 44 \\
\hline Fold Axis & 40.415985 & 22.228492 & 0 & 209 \\
\hline Fold Axis & 40.416256 & 22.229741 & 9 & 140 \\
\hline Fold Axis & 40.416461 & 22.232528 & 2 & 285 \\
\hline Fold Axis & 40.416740 & 22.233087 & 15 & 313 \\
\hline Fold Axis & 40.416821 & 22.233334 & 62 & 307 \\
\hline Fold Axis & 40.416919 & 22.233359 & 7 & 139 \\
\hline Fold Axis & 40.416903 & 22.233385 & 7 & 138 \\
\hline Stretching Lineation & 40.455869 & 22.249521 & 0 & 39 \\
\hline Stretching Lineation & 40.456822 & 22.250093 & 10 & 30 \\
\hline Stretching Lineation & 40.456822 & 22.250093 & 6 & 29 \\
\hline Stretching Lineation & 40.456820 & 22.250113 & 13 & 31 \\
\hline Stretching Lineation & 40.456828 & 22.250099 & 13 & 26 \\
\hline Stretching Lineation & 40.456846 & 22.250120 & 11 & 14 \\
\hline Stretching Lineation & 40.456856 & 22.250132 & 16 & 19 \\
\hline Stretching Lineation & 40.456855 & 22.250135 & 6 & 29 \\
\hline Stretching Lineation & 40.456849 & 22.250157 & 9 & 27 \\
\hline Stretching Lineation & 40.455801 & 22.222052 & 28 & 202 \\
\hline Stretching Lineation & 40.455860 & 22.222059 & 9 & 6 \\
\hline Stretching Lineation & 40.479272 & 22.253991 & 17 & 239 \\
\hline Stretching Lineation & 40.437309 & 22.175778 & 5 & 222 \\
\hline Stretching Lineation & 40.430029 & 22.187984 & 3 & 215 \\
\hline Stretching Lineation & 40.456903 & 22.250139 & 15 & 28 \\
\hline Stretching Lineation & 40.456903 & 22.250139 & 7 & 23 \\
\hline Stretching Lineation & 40.456905 & 22.250154 & 11 & 19 \\
\hline Stretching Lineation & 40.437490 & 22.247828 & 11 & 214 \\
\hline Stretching Lineation & 40.437739 & 22.246829 & 4 & 175 \\
\hline Stretching Lineation & 40.432548 & 22.239776 & 66 & 226 \\
\hline Stretching Lineation & 40.432457 & 22.240026 & 18 & 208 \\
\hline
\end{tabular}


Supplementary Data Table S3

\begin{tabular}{|c|c|c|c|c|}
\hline Stretching Lineation & 40.432416 & 22.239913 & 13 & 203 \\
\hline Stretching Lineation & 40.432413 & 22.240091 & 17 & 210 \\
\hline Stretching Lineation & 40.426812 & 22.240810 & 15 & 62 \\
\hline Stretching Lineation & 40.426311 & 22.241185 & 16 & 40 \\
\hline Stretching Lineation & 40.425613 & 22.240841 & 5 & 231 \\
\hline Stretching Lineation & 40.424980 & 22.240654 & 14 & 223 \\
\hline Stretching Lineation & 40.423812 & 22.240936 & 16 & 55 \\
\hline Stretching Lineation & 40.442936 & 22.259149 & 17 & 8 \\
\hline Stretching Lineation & 40.443815 & 22.262139 & 18 & 317 \\
\hline Stretching Lineation & 40.444561 & 22.261392 & 6 & 141 \\
\hline Stretching Lineation & 40.444599 & 22.261095 & 6 & 137 \\
\hline Stretching Lineation & 40.444718 & 22.260831 & 51 & 95 \\
\hline Stretching Lineation & 40.444783 & 22.260877 & 7 & 177 \\
\hline Stretching Lineation & 40.444191 & 22.260001 & 20 & 50 \\
\hline Stretching Lineation & 40.444191 & 22.259968 & 45 & 255 \\
\hline Stretching Lineation & 40.417294 & 22.245934 & 16 & 190 \\
\hline Stretching Lineation & 40.417307 & 22.245926 & 0 & 42 \\
\hline Stretching Lineation & 40.418734 & 22.244298 & 6 & 236 \\
\hline Stretching Lineation & 40.418921 & 22.244171 & 10 & 50 \\
\hline Stretching Lineation & 40.418888 & 22.244170 & 4 & 228 \\
\hline Stretching Lineation & 40.418750 & 22.242218 & 18 & 238 \\
\hline Stretching Lineation & 40.418537 & 22.240503 & 12 & 243 \\
\hline Stretching Lineation & 40.416091 & 22.226759 & 23 & 63 \\
\hline Stretching Lineation & 40.415383 & 22.229338 & 0 & 40 \\
\hline Stretching Lineation & 40.416375 & 22.229689 & 3 & 30 \\
\hline Stretching Lineation & 40.416437 & 22.232554 & 2 & 201 \\
\hline Stretching Lineation & 40.417054 & 22.233558 & 33 & 225 \\
\hline Stretching Lineation & 40.417076 & 22.233573 & 9 & 31 \\
\hline Stretching Lineation & 40.418184 & 22.237363 & 12 & 27 \\
\hline Stretching Lineation & 40.471549 & 22.211449 & 8 & 77 \\
\hline Stretching Lineation & 40.473233 & 22.210659 & 0 & 40 \\
\hline Stretching Lineation & 40.477391 & 22.216691 & 27 & 61 \\
\hline Mineral Lineation & 40.425521 & 22.186178 & 18 & 175 \\
\hline Mineral Lineation & 40.393502 & 22.209526 & 12 & 352 \\
\hline Mineral Lineation & 40.467740 & 22.243037 & 33 & 241 \\
\hline Mineral Lineation & 40.437967 & 22.175508 & 21 & 246 \\
\hline Mineral Lineation & 40.422718 & 22.240286 & 2 & 30 \\
\hline Slickenside & 40.420695 & 22.193305 & 51 & 69 \\
\hline Slickenside & 40.425449 & 22.190030 & 34 & 354 \\
\hline Slickenside & 40.461977 & 22.247124 & 57 & 331 \\
\hline Slickenside & 40.412195 & 22.196075 & 70 & 258 \\
\hline Slickenside & 40.441853 & 22.244594 & 25 & 313 \\
\hline Slickenside & 40.444718 & 22.260850 & 61 & 157 \\
\hline Slickenside & 40.480622 & 22.219532 & 6 & 235 \\
\hline Slickenside & 40.480649 & 22.219570 & 5 & 304 \\
\hline Slickenside & 40.479698 & 22.219041 & 50 & 234 \\
\hline Slickenside & 40.479703 & 22.218986 & 31 & 255 \\
\hline Slickenside & 40.479804 & 22.219360 & 35 & 251 \\
\hline
\end{tabular}


Supplementary Data

Table S3 
Table S3: Table of structural measurements taken in mapped area. 
age :age (Ma)

ClL :lower 0.95 confidential interval (Ma)

clU :upper 0.95 confidential interval (Ma)

$\mathrm{sE}$ :standard error (Ma)

$\begin{array}{ccccc} & \text { age } & \text { clL } & \text { clU } & \text { sE } \\ \text { Pooled } & 157 & 148 & 167.66 & 5 \\ \text { Central } & 156 & 138 & 176.74 & 10\end{array}$

Probability of $\chi 2(\%): \quad 0$

Age dispersion (\%): $\quad 23.88$

Pooled spontaneous tracks: $\quad 5628$

Pooled induced tracks: $\quad 1353$

Pooled counter squares: $\quad 46034$

Mean U concentration +/- $1 \sigma(\mathrm{ppm}): 222+/-13$

SINGLE GRAIN DATA in ORIGINAL ORDER:

$\mathrm{n}$ :grain number

$\mathrm{mN}$ :mount number

gNM :grain number per mount

nS :spontaneous tracks (tr)

rhos :density of spontaneous tracks $\left(\mathrm{tr} / \mathrm{cm}^{-2}\right)$

$\mathrm{nl}$ :induced tracks

rhol :density of induced tracks $\left(\operatorname{tr} / \mathrm{cm}^{-2}\right)$

$\mathrm{nSq}$ :number of counter squares

uG :uranium concentration (ppm)

UGSE :standard error of uranium concentration

age : grain age (Ma)

CILG :lower 0.95 confidential interval (Ma)

clUG :upper 0.95 confidential interval (Ma)

sEG :relative standard error $(1 \sigma, \mathrm{Ma})$

... in ORIGINAL ORDER:

\begin{tabular}{|c|c|c|c|c|c|c|c|c|c|c|c|c|c|}
\hline \multicolumn{3}{|c|}{$\mathrm{mN}$ gNM } & $\mathrm{nS}$ & rhos & $\mathrm{nl}$ & rhol & $\mathrm{nSq}$ & uG & uGSE & ageG & clLG & clUG & sEG \\
\hline 1 & 1 & 1 & 39 & $1.14 \mathrm{E}+07$ & 10 & $2.92 \mathrm{E}+06$ & 343 & 219 & 135 & 146.5 & 73.08 & 328.53 & 49.86 \\
\hline 2 & 1 & 2 & 77 & $1.13 E+C$ & 21 & $3.09 \mathrm{E}+06$ & 679 & 233 & 10 & 138.7 & 85.5 & 00.0 & J. \\
\hline & 1 & 3 & 85 & $56 \mathrm{E}+07$ & 21 & $.85 E+06$ & 546 & 289 & 12 & 152 & 9 & & \\
\hline & 1 & 4 & 4 & $E+07$ & 17 & 6 & 91 & 440 & & 8 & 1.32 & & \\
\hline & 1 & 5 & 49 & $E+07$ & 20 & 66 & 312 & 482 & 21 & 93.6 & 4.72 & & \\
\hline & 1 & 6 & 60 & $1.11 \mathrm{E}+07$ & 13 & $40 \mathrm{E}+06$ & 541 & 181 & 9 & 173.4 & 5.6 & & \\
\hline & 1 & 7 & 77 & $1.33 \mathrm{E}+07$ & 13 & $2.24 \mathrm{E}+06$ & 581 & 168 & 92 & 221.6 & 124.4 & 432.39 & F.J \\
\hline & 1 & 8 & 41 & $9.88 \mathrm{E}+06$ & 16 & $3.86 \mathrm{E}+06$ & 415 & 290 & 143 & 97 & 53.87 & & .5 \\
\hline & 1 & 9 & 57 & $7.33 \mathrm{E}+06$ & 17 & $2.19 \mathrm{E}+$ & 778 & 164 & 7 & & & & \\
\hline & 1 & 10 & 85 & & 16 & & & 270 & 13 & & & & \\
\hline & 2 & 1 & 44 & & 9 & & 34 & 186 & 12 & 18 & & & 3.7 \\
\hline & 2 & 2 & 55 & 1.7 & 9 & & 315 & 215 & 14 & 27 & & & .86 \\
\hline & 2 & 3 & 25 & 7.72 & 5 & & & 1 & & & & & \\
\hline & 2 & 4 & 123 & 1.2 & 41 & & & 312 & 9 & & & & \\
\hline & 2 & 5 & 66 & $1.12 \mathrm{E}+\mathrm{C}$ & 17 & & 587 & 218 & 10 & & 85.78 & & \\
\hline & 2 & 6 & 40 & $1.28 \mathrm{E}+07$ & 8 & $2.56 \mathrm{E}+$ & 313 & 193 & 13 & 186.1 & 87.97 & 458.05 & 68.4 \\
\hline & 2 & 7 & 69 & $1.57 \mathrm{E}+07$ & 30 & $6.80 \mathrm{E}+$ & & 513 & 18 & 87.4 & 56.44 & 138.96 & 18.8 \\
\hline & 2 & 8 & 58 & $9.34 \mathrm{E}+06$ & 13 & $2.09 \mathrm{E}+06$ & 621 & 158 & 8 & 167.4 & 92.02 & 332.04 & 99.76 \\
\hline & 2 & 9 & 61 & $1.12 E+07$ & 15 & $2.76 \mathrm{E}+06$ & 543 & 208 & 106 & 153 & 87 & 289.18 & 42.9 \\
\hline
\end{tabular}




\begin{tabular}{|c|c|c|c|c|c|c|c|c|c|c|c|c|}
\hline & 17 & 41 & $8.93 E+06$ & 13 & $2.83 \mathrm{E}+06$ & 459 & 214 & 116 & 118.9 & 63.24 & 241.67 & 36.69 \\
\hline & 18 & 62 & $1.87 E+07$ & 22 & $65 E+06$ & 331 & 501 & & & & 182.25 & 26.02 \\
\hline & 19 & 73 & $.07 \mathrm{E}+07$ & 29 & $.26 \mathrm{E}+06$ & 681 & 321 & & & & & \\
\hline & 1 & 105 & $79 \mathrm{E}+07$ & 27 & $4.60 \mathrm{E}+06$ & 587 & 348 & & & & & \\
\hline & 2 & 88 & $46 \mathrm{E}+07$ & 20 & 06 & 605 & 250 & & & & & 12 \\
\hline & 3 & 47 & $00 \mathrm{E}+07$ & 12 & $55 E+06$ & 470 & 93 & & & & & $-\infty$ \\
\hline & 4 & 67 & $4 \mathrm{E}+07$ & 30 & $91 \mathrm{E}+06$ & 434 & 22 & & & & & \\
\hline & 5 & 60 & $21 \mathrm{E}+07$ & 31 & $25 E+06$ & 496 & 72 & & & & & \\
\hline & 6 & 96 & $.51 \mathrm{E}+07$ & 23 & $.01 \mathrm{E}+06$ & 383 & 454 & 187 & 157 & 9.74 & 258.92 & 5.8 \\
\hline & 7 & 45 & $7.56 \mathrm{E}+06$ & 13 & +06 & 595 & 165 & & 130.1 & 9.86 & & 39.7 \\
\hline & 8 & 90 & $1.20 \mathrm{E}+07$ & 36 & +06 & 748 & 364 & 12 & 94.77 & & & 18.5 \\
\hline & 9 & 195 & $49 \mathrm{E}+07$ & 55 & $E+06$ & 1310 & 317 & & 134.1 & & & 20.36 \\
\hline & 10 & 57 & $1.07 \mathrm{E}+07$ & 22 & $E+06$ & 533 & 312 & & 01 & & & 24.17 \\
\hline & 1 & 67 & +07 & 22 & & 337 & 493 & & & & & 7.76 \\
\hline & 12 & 54 & +07 & 14 & & 330 & 321 & & & & & 42.18 \\
\hline & 13 & 66 & +07 & 18 & 06 & 229 & 94 & 27 & & & & $0.8 \xi$ \\
\hline & 14 & 50 & +07 & 16 & & 332 & 64 & $17 \subseteq$ & & & & 2.95 \\
\hline & 15 & 24 & +06 & 4 & 55 & 548 & & 5 & 218.6 & & & 106.5 \\
\hline & 16 & 66 & +07 & 11 & 06 & 573 & 14 & 8 & 223 & & & 69.8 \\
\hline & 17 & 112 & +07 & 19 & & 900 & & 7 & 220.3 & & & \\
\hline & 18 & 120 & +07 & 12 & & 588 & & 8 & & & & \\
\hline & 19 & 64 & +07 & 9 & & 373 & 18 & & & & & \\
\hline & 3 & 94 & & 31 & & 907 & & & & & & \\
\hline & 3 & 78 & & 17 & & 36 & & & & & & \\
\hline & 22 & 89 & & 16 & & 538 & & & & & & \\
\hline & 23 & 63 & 07 & 13 & & 388 & & & & & & \\
\hline & 2 & 94 & +07 & 23 & & 577 & & & & & & \\
\hline & 25 & 38 & +06 & 10 & & 411 & 18 & 11 & 2.2 & & & 3.5 \\
\hline & 26 & 43 & +06 & 6 & & 558 & & 6 & 62.8 & & & 106.6 \\
\hline & 27 & 53 & +06 & 14 & & 928 & & & & & & \\
\hline & 4 & 47 & +07 & 18 & & 335 & & & & & & \\
\hline & 4 & & & 8 & & & & & & & & \\
\hline & 4 & 49 & & 10 & & 37 & & & & & & \\
\hline & 4 & & & 13 & & 8 & & & & & & \\
\hline & 5 & 208 & & 29 & & 1186 & & & & & & 2 \\
\hline & 6 & 47 & +07 & 12 & & 383 & 23 & & & & & 45.79 \\
\hline & 7 & 74 & +07 & 17 & & 364 & 35 & 16 & & & & 42.8 \\
\hline & 8 & 83 & +06 & 1 & & 1028 & 12 & & & & & 47.43 \\
\hline & 9 & 110 & +07 & 13 & & 648 & & & & & & 88.51 \\
\hline & 4 & 78 & +07 & 21 & & 352 & & & & & & 3.67 \\
\hline & 1 & 54 & & 13 & & & 22 & & & & & \\
\hline & 4 & 106 & & 17 & & & 75 & & & & & 59.15 \\
\hline & 4 & 161 & & 33 & & & 248 & & & & & 34.58 \\
\hline & 1 & 37 & & 4 & & & & & & & & \\
\hline & 1 & 40 & & 3 & & & & & & & & \\
\hline & 1 & 79 & & 15 & & & & & & & & \\
\hline & 1 & 88 & & 16 & & & 12 & 7 & & & .82 & 54.31 \\
\hline & 1 & 7 & & 14 & & & 19 & 10 & & & & 4.38 \\
\hline & 1 & 41 & & 9 & & & & & & & & \\
\hline & 2 & 87 & & 21 & & 1128 & 14 & & & & & 708 \\
\hline & 4 & 98 & & 12 & & & 16 & 9 & & & & \\
\hline 15 & 22 & 64 & $1.05 \mathrm{E}+07$ & 12 & $1.96 \mathrm{E}+06$ & 612 & 148 & 84 & 198.5 & 108 & 402.16 & 60.29 \\
\hline & 23 & 49 & $1.33 \mathrm{E}+07$ & 10 & $2.72 \mathrm{E}+06$ & 368 & 206 & 127 & 182.2 & 92.98 & 401.89 & 60.66 \\
\hline
\end{tabular}




\begin{tabular}{|c|c|c|c|c|c|c|c|c|c|c|c|c|c|}
\hline 4 & & 4 & 41 & $1.41 \mathrm{E}+07$ & 17 & $5.84 \mathrm{E}+06$ & 291 & 440 & 210 & 91.58 & 51.32 & 171.89 & \\
\hline 5 & 1 & 5 & 49 & $1.57 \mathrm{E}+07$ & 20 & $6.41 \mathrm{E}+06$ & 312 & 482 & 213 & & & & \\
\hline 37 & & 8 & 90 & $1.20 \mathrm{E}+07$ & 36 & $4.81 \mathrm{E}+06$ & 748 & 364 & 121 & & & & \\
\hline 29 & & 19 & 73 & $1.07 \mathrm{E}+07$ & 29 & $4.26 \mathrm{E}+06$ & 681 & 321 & 118 & 95.56 & 1.71 & & 0.7 \\
\hline 8 & 1 & 8 & 41 & $9.88 \mathrm{E}+06$ & 16 & $3.86 \mathrm{E}+06$ & 415 & 290 & 143 & 97.2 & 3.87 & & 95 \\
\hline 39 & 3 & 10 & 57 & $E+07$ & 22 & $\mathrm{E}+06$ & 533 & 312 & 132 & 98.01 & & & \\
\hline & & 1 & 47 & $1.40 \mathrm{E}+07$ & 18 & $7 E+06$ & 335 & 407 & 189 & & & & \\
\hline & & 10 & 42 & $1.01 \mathrm{E}+07$ & 5 & $E+06$ & 415 & 273 & 139 & & & & \\
\hline & & 12 & 31 & $E+06$ & 1 & $3 E+06$ & 569 & 146 & 86 & & & & \\
\hline & & 18 & 62 & $E+07$ & 22 & $6.65 E+06$ & 331 & 501 & 212 & 106.7 & & & \\
\hline 14 & 2 & 4 & 123 & $1.24 \mathrm{E}+07$ & 41 & $4.14 \mathrm{E}+06$ & 990 & 312 & 97 & 3.8 & 79.7 & .15 & \\
\hline 49 & 3 & 20 & 94 & $=+07$ & 31 & $E+06$ & 907 & 258 & 92 & 114.7 & .14 & & \\
\hline 0 & 3 & 11 & 67 & $E+07$ & 22 & $E+06$ & 337 & 493 & 208 & & & & \\
\hline 3 & & 14 & 50 & +07 & 16 & $E+06$ & 332 & 364 & 179 & & & & \\
\hline 27 & 2 & 17 & 41 & 8.9 & 13 & +06 & 459 & 214 & 116 & & & & \\
\hline 9 & 1 & 9 & 57 & 06 & 17 & +06 & 778 & 16 & 79 & & & & \\
\hline 24 & & 14 & 41 & +06 & 2 & +06 & 577 & 15 & 89 & & & & \\
\hline 6 & & 7 & 45 & 06 & 3 & +06 & 595 & 16 & 9 & & & & \\
\hline 8 & 3 & 9 & 195 & +07 & 5 & +06 & 1310 & 317 & 85 & 4.1 & & & \\
\hline 42 & 3 & 13 & 66 & 2.88 & 18 & $E+06$ & 229 & 59 & 276 & 138 & .81 & & \\
\hline 2 & 1 & 2 & 77 & +07 & 1 & $E+06$ & 679 & 23 & 100 & & & & \\
\hline 66 & 4 & 10 & 78 & 2.2 & 21 & $E+06$ & 352 & 452 & 195 & & & & \\
\hline 6 & 3 & 27 & 53 & +06 & 14 & $E+06$ & 928 & 114 & 60 & & & & \\
\hline 54 & 3 & 25 & 38 & & 10 & & 411 & 184 & & & & & \\
\hline 1 & 3 & 12 & 54 & & 14 & & 33 & 32 & & & & & \\
\hline 15 & 2 & 5 & 66 & & 17 & +06 & 587 & 21 & 10 & & & & \\
\hline 62 & & 6 & 47 & & 12 & +06 & 383 & 23 & & & & & \\
\hline 1 & 1 & 1 & 39 & & 10 & +06 & 343 & 21 & & & & & \\
\hline 30 & & 1 & 105 & 1.7 & & +06 & 587 & 34 & 13 & & & & \\
\hline 32 & & 3 & 47 & 1.0 & 12 & & 47 & 19 & 10 & & & & \\
\hline 3 & . & 3 & 85 & & 21 & +06 & 546 & 28 & 12 & & & & \\
\hline 19 & 2 & 9 & 61 & & 15 & +06 & 543 & 208 & 106 & & & & \\
\hline 53 & 3 & 24 & 94 & & 23 & & 577 & 301 & 124 & & & & 35.1 \\
\hline 67 & 4 & 11 & 54 & & 13 & & & 229 & 125 & & & & \\
\hline 6 & 2 & 20 & 87 & & 21 & & 11 & 14 & & & & & \\
\hline 2 & & 16 & 79 & & 19 & & & & & & & & \\
\hline 35 & 3 & 6 & 96 & & 23 & +06 & 383 & 45 & 18 & & & & 35 \\
\hline 21 & 2 & 11 & 189 & & 44 & +06 & 938 & 35 & 10 & & & & $26 . \varsigma$ \\
\hline 63 & 4 & 7 & 74 & 07 & 1 & +06 & 364 & 35 & 16 & & & & \\
\hline 31 & 3 & 2 & 88 & & 20 & & 605 & 25 & & & & & \\
\hline 18 & 2 & 8 & 58 & +06 & 13 & +06 & 621 & 158 & 86 & & & & \\
\hline 75 & 4 & 19 & 41 & & 9 & & 799 & 85 & 55 & & & & 59.5 \\
\hline 50 & 3 & 21 & 78 & & 17 & & 636 & 202 & 97 & & & & \\
\hline 6 & 1 & 6 & 60 & & 13 & & & & & & & & \\
\hline & 3 & 23 & 63 & & 13 & & 388 & 25 & & & & & \\
\hline & 4 & 3 & 49 & & 10 & & 337 & 22 & & & & & \\
\hline & 4 & 23 & 49 & & 10 & & 368 & 20 & 127 & & & & \\
\hline 11 & 2 & 1 & 44 & & 9 & & 364 & 18 & 121 & & & & \\
\hline & 4 & 8 & 83 & & 17 & +06 & 1028 & 12 & 60 & & & & \\
\hline 23 & 2 & 13 & 49 & & 10 & $E+06$ & 508 & 14 & 92 & & 38 & & 60.5 \\
\hline 69 & 4 & 13 & 161 & $1.60 \mathrm{E}+07$ & 33 & $3.27 \mathrm{E}+06$ & 1009 & 24 & 86 & & 126.2 & 274.23 & \\
\hline 13 & 2 & 3 & 25 & $7.72 \mathrm{E}+06$ & 5 & $1.54 \mathrm{E}+06$ & 324 & 116 & 99 & & 71.73 & 611.93 & 83.2 \\
\hline & 2 & 6 & 40 & $1.28 \mathrm{E}+07$ & 8 & $2.56 \mathrm{E}+06$ & 313 & 193 & 132 & 186.1 & 87.97 & 458.05 & 68.4 \\
\hline
\end{tabular}




$\begin{array}{cccccccccccccc}7 & 1 & 7 & 77 & 1.33 \mathrm{E}+07 & 13 & 2.24 \mathrm{E}+06 & 581 & 168 & 92 & 221.6 & 124.4 & 432.39 & 64.32 \\ 25 & 2 & 15 & 54 & 7.51 \mathrm{E}+06 & 9 & 1.25 \mathrm{E}+06 & 719 & 94 & 61 & 222.9 & 111.7 & 509.9 & 76.58 \\ 45 & 3 & 16 & 66 & 1.15 \mathrm{E}+07 & 11 & 1.92 \mathrm{E}+06 & 573 & 145 & 86 & 223 & 119.3 & 465.42 & 69.89 \\ 12 & 2 & 2 & 55 & 1.75 \mathrm{E}+07 & 9 & 2.86 \mathrm{E}+06 & 315 & 215 & 140 & 227 & 113.9 & 518.52 & 77.86 \\ 68 & 4 & 12 & 106 & 6.18 \mathrm{E}+06 & 17 & 9.91 \mathrm{E}+05 & 1716 & 75 & 36 & 232.1 & 140.4 & 411.03 & 59.15 \\ 48 & 3 & 19 & 64 & 1.72 \mathrm{E}+07 & 9 & 2.41 \mathrm{E}+06 & 373 & 182 & 118 & 262.7 & 133.4 & 594.36 & 89.17 \\ 55 & 3 & 26 & 43 & 7.71 \mathrm{E}+06 & 6 & 1.08 \mathrm{E}+06 & 558 & 81 & 64 & 262.8 & 115.2 & 744.45 & 106.69 \\ 61 & 4 & 5 & 208 & 1.75 \mathrm{E}+07 & 29 & 2.45 \mathrm{E}+06 & 1186 & 185 & 68 & 267.1 & 182.5 & 406.5 & 52.21 \\ 77 & 4 & 21 & 98 & 1.80 \mathrm{E}+07 & 12 & 2.21 \mathrm{E}+06 & 544 & 167 & 95 & 301.2 & 168.5 & 596.27 & 88.84 \\ 65 & 4 & 9 & 110 & 1.70 \mathrm{E}+07 & 13 & 2.01 \mathrm{E}+06 & 648 & 152 & 83 & 312.1 & 178.9 & 598.03 & 88.51 \\ 60 & 4 & 4 & 114 & 1.31 \mathrm{E}+07 & 13 & 1.50 \mathrm{E}+06 & 869 & 113 & 62 & 323.1 & 185.5 & 618.13 & 91.46 \\ 70 & 4 & 14 & 37 & 7.05 \mathrm{E}+06 & 4 & 7.62 \mathrm{E}+05 & 525 & 58 & 54 & 332.5 & 125.7 & 1238.42 & 157.36 \\ 47 & 3 & 18 & 120 & 2.04 \mathrm{E}+07 & 12 & 2.04 \mathrm{E}+06 & 588 & 154 & 87 & 367.6 & 207.8 & 720.28 & 107.28 \\ 71 & 4 & 15 & 40 & 1.55 \mathrm{E}+07 & 3 & 1.16 \mathrm{E}+06 & 258 & 88 & 94 & 468.3 & 160.2 & 2166.27 & 243.06\end{array}$

PARAMETERS:

nM: Number of mounts

rhoD: Effective track density $\left(\mathrm{tr} / \mathrm{cm}^{2}\right)$

$\mathrm{nD}$ : Count for fluence monitor (tr)

uGlass: Uranium concentration of glass standard (ppm)

zeta: zeta factor $\left(\mathrm{a} \mathrm{cm}^{2} / \mathrm{tr}\right)$

zetaSE: standard error of zeta factor $\left(a \mathrm{~cm}^{2} / \mathrm{tr}\right)$

sSC: size of square counter $\left(\mathrm{cm}^{2}\right)$

$\begin{array}{rcc}\text { nM } & \text { rhoD } & \text { nD } \\ 1 & 5.29 \mathrm{E}+05 & 6594 \\ 2 & 5.28 \mathrm{E}+05 & 6579 \\ 3 & 5.27 \mathrm{E}+05 & 6565 \\ 4 & 5.26 \mathrm{E}+05 & 6551\end{array}$

$\begin{array}{rc}\text { uGlass } & \text { zeta } \\ 39.8 & 145.39 \\ 39.8 & 145.39 \\ 39.8 & 145.39 \\ 39.8 & 145.39\end{array}$

zetaSE
7.04
7.04
7.04
7.04

SSC

$1.0 \mathrm{E}-08$

$1.0 \mathrm{E}-08$

$1.0 \mathrm{E}-08$

$1.0 \mathrm{E}-08$

Sample ID

v1504 a-c, ETH404, \#7-8, GF 43013

SAMPLE POOLED and CENTRAL AGES

age :age (Ma)

ClL :lower 0.95 confidential interval (Ma)

clU :upper 0.95 confidential interval (Ma)

$\mathrm{sE}$ :standard error (Ma)

$\begin{array}{ccccc} & \text { age } & \text { clL } & \text { clU } & \text { sE } \\ \text { Pooled } & 177 & 165 & 189.65 & 6 \\ \text { Central } & 177 & 153 & 204.12 & 13\end{array}$

Probability of $\chi 2(\%): \quad 0$

Age dispersion (\%): $\quad 34.45$

Pooled spontaneous tracks: $\quad 5101$

Pooled induced tracks: $\quad 1083$

Pooled counter squares: $\quad 38078$

Mean U concentration +/- $1 \sigma(\mathrm{ppm}): 216+/-14$

SIN GRDAT/ in ORIGINAL ORDER: 
UG :uranium concentration (ppm)

UGSE :standard error of uranium concentration age $:$ grain age $(\mathrm{Ma})$

cILG :lower 0.95 confidential interval (Ma)

clUG :upper 0.95 confidential interval (Ma)

sEG :relative standard error $(1 \sigma, \mathrm{Ma})$

... in ORIGINAL ORDER:

\begin{tabular}{|c|c|c|c|c|c|c|c|c|c|c|c|c|c|}
\hline \multicolumn{3}{|c|}{$\mathrm{mN}$ gNM } & $\mathrm{nS}$ & rhos & $\mathrm{nl}$ & rhol & $\mathrm{nSq}$ & uG & uGSE & ageG & CILG & clUG & sEG \\
\hline 1 & 1 & 1 & 67 & $1.38 \mathrm{E}+07$ & 19 & $3.93 \mathrm{E}+06$ & 484 & 299 & 135 & 132 & 79.11 & 232.36 & 33.58 \\
\hline 2 & 1 & 2 & 86 & $2.58 \mathrm{E}+07$ & 15 & $4.49 \mathrm{E}+06$ & 334 & 342 & 174 & 212.6 & 123.9 & & \\
\hline J & 1 & 3 & 32 & $8.84 \mathrm{E}+06$ & 9 & $49 \mathrm{E}+06$ & 362 & 189 & 123 & 132 & & & \\
\hline & 1 & 4 & 139 & & 48 & 6 & 252 & 162 & 47 & 10 & & & \\
\hline & 1 & 5 & & $E+07$ & 19 & & 449 & 322 & 146 & 137.8 & & 2 & \\
\hline & 1 & 6 & 35 & $E+07$ & & & 347 & & 83 & & & & \\
\hline$r$ & 1 & 7 & 79 & $0 \mathrm{E}+07$ & 15 & & 226 & 505 & 257 & 195.6 & & & \\
\hline 8 & 1 & 8 & 51 & 04E+07 & 11 & +06 & 492 & 170 & 100 & 172 & 0.12 & & \\
\hline 9 & 1 & 9 & 123 & $81 \mathrm{E}+07$ & 29 & 06 & 438 & 504 & 186 & 158.7 & 106 & & \\
\hline & 1 & 10 & 107 & $63 \mathrm{E}+07$ & 35 & $33 \mathrm{E}+06$ & 657 & 405 & 136 & 114.9 & & & \\
\hline & 1 & 11 & 71 & $1.35 \mathrm{E}+07$ & 18 & & 528 & 259 & 121 & & & & \\
\hline 2 & 1 & 12 & 58 & & 15 & & 497 & 230 & 117 & & & & \\
\hline & 1 & 13 & 9 & & 34 & & 07 & 426 & & & & & \\
\hline & 1 & 14 & & & 20 & & & 37 & & & & & \\
\hline & 1 & 15 & & & 12 & & & & 9 & & & & \\
\hline 6 & 1 & 16 & & $E+07$ & 20 & & 412 & 309 & 16 & 3 & & & \\
\hline 1 & 1 & 17 & 61 & $4 \mathrm{E}+07$ & 21 & 06 & 372 & 429 & 18 & 10 & 5.98 & & 27.07 \\
\hline 18 & 1 & $1 \varepsilon$ & 66 & $5 \mathrm{E}+07$ & 10 & & 356 & 214 & 132 & 24 & 127 & & \\
\hline 9 & 2 & 1 & 34 & $6.98 \mathrm{E}+06$ & 9 & & 487 & & 91 & & & & \\
\hline 0 & 2 & 2 & 6 & $6.89 \mathrm{E}+06$ & 19 & & 885 & & 72 & & & & \\
\hline & 2 & 3 & & & & & & & & & & & \\
\hline 2 & 2 & 4 & 149 & & 29 & & & 239 & 88 & & & & \\
\hline 3 & 2 & 5 & 133 & & 36 & & & & 76 & & & & \\
\hline & 2 & 6 & 5 & & 19 & & & & & & & & \\
\hline & 2 & 7 & 8 & & 13 & & 444 & 222 & & & & & \\
\hline & 2 & 8 & 7 & & 1 & & & & & & & & \\
\hline & 2 & 9 & 8 & +07 & 1 & & & 2 & & & & & \\
\hline & 2 & & 6 & & & & & & & & & & \\
\hline & 2 & 1 & 2 & 7 & & & & 26 & & & & & \\
\hline & 2 & 1 & & & & & & & & & & & \\
\hline & 2 & 1 & & & & & & & & & & & \\
\hline & 2 & 1 & & & 1 & & & 2 & & & & & \\
\hline & 2 & 1 & 8 & & 1 & & & & & & & & \\
\hline & 2 & 1 & & & & & & & & & & & \\
\hline & 2 & 1 & & & 14 & & & 23 & 12 & & & & \\
\hline & 2 & 1 & 10 & -07 & 8 & $1.74 \mathrm{E}$ & 461 & 1 & 90 & 7 & & 1073.15 & $161+2$ \\
\hline & 2 & 1 & 8 & $28 \mathrm{E}+07$ & 5 & & & 1 & 8 & & & 0.38 & \\
\hline & 2 & 2 & 101 & $94 \mathrm{E}+07$ & 8 & & & 1 & & & & 4.42 & \\
\hline & 2 & 2 & 68 & $2.13 \mathrm{E}+07$ & 16 & $\mathrm{O} 00 \mathrm{~F}+$ & 320 & 37 & 187 & & 92.28 & 71 & \\
\hline 0 & 2 & 2 & 36 & & 8 & & & & & & & & \\
\hline & 2 & 23 & 97 & $1.73 \mathrm{E}+07$ & 19 & $3.39 E+06$ & 561 & 257 & & & & 3.77 & 46.76 \\
\hline & 2 & 24 & 64 & $1.52 \mathrm{E}+07$ & 16 & & $4 \angle 1$ & 28 & & & & & 7.1 \\
\hline & 2 & 25 & 76 & $1.55 \mathrm{E}+07$ & 16 & & 492 & 247 & 122 & & & & \\
\hline & 2 & 26 & 86 & $2.82 \mathrm{E}+07$ & 0 & $2.0 \angle \Sigma+00$ & 305 & 199 & 137 & 390.4 & 195.9 & 912.19 & \\
\hline
\end{tabular}




$\begin{array}{cccccccccccccc}52 & 2 & 34 & 191 & 1.67 \mathrm{E}+07 & 14 & 1.23 \mathrm{E}+06 & 1142 & 93 & 49 & 495 & 295.5 & 903.49 & 132.73 \\ 53 & 2 & 35 & 120 & 7.83 \mathrm{E}+06 & 29 & 1.89 \mathrm{E}+06 & 1532 & 144 & 53 & 155.2 & 103.6 & 241.12 & 31.69 \\ 54 & 2 & 36 & 93 & 1.80 \mathrm{E}+07 & 13 & 2.52 \mathrm{E}+06 & 517 & 191 & 104 & 264.3 & 150.1 & 510.78 & 75.72 \\ 55 & 2 & 37 & 31 & 5.85 \mathrm{E}+06 & 10 & 1.89 \mathrm{E}+06 & 530 & 143 & 88 & 115.9 & 56.21 & 264.82 & 40.47 \\ 56 & 2 & 38 & 97 & 1.42 \mathrm{E}+07 & 26 & 3.81 \mathrm{E}+06 & 682 & 289 & 113 & 140.1 & 90.79 & 224.69 & 30.47 \\ 57 & 2 & 39 & 130 & 2.17 \mathrm{E}+07 & 9 & 1.51 \mathrm{E}+06 & 598 & 114 & 74 & 520 & 275.3 & 1127.19 & 170.45 \\ 58 & 2 & 40 & 93 & 1.33 \mathrm{E}+07 & 16 & 2.28 \mathrm{E}+06 & 701 & 173 & 85 & 216.1 & 128.2 & 391.7 & 56.97 \\ 59 & 2 & 41 & 42 & 1.12 \mathrm{E}+07 & 4 & 1.06 \mathrm{E}+06 & 376 & 81 & 76 & 375.2 & 143.6 & 1378.86 & 176.33 \\ 60 & 2 & 42 & 97 & 1.70 \mathrm{E}+07 & 35 & 6.14 \mathrm{E}+06 & 570 & 466 & 157 & 104.5 & 70.69 & 158.41 & 20.39 \\ 61 & 2 & 43 & 243 & 1.16 \mathrm{E}+07 & 72 & 3.43 \mathrm{E}+06 & 2100 & 260 & 61 & 126.8 & 97.31 & 165.11 & 17.27\end{array}$

\section{NCREASING AGE}

\begin{tabular}{|c|c|c|c|c|c|c|c|c|c|c|c|c|c|}
\hline \multicolumn{3}{|c|}{$\mathrm{mN}$ gNM } & nS & rhos & $\mathrm{nl}$ & rhol & $n S q$ & uG & uGSE & ageG & clLG & clUG & sEG \\
\hline 29 & 2 & 11 & 24 & $7.04 \mathrm{E}+06$ & 12 & $3.52 \mathrm{E}+06$ & 341 & 267 & 151 & 75.3 & 36.56 & 165.25 & 25.74 \\
\hline 2 & 2 & 14 & 49 & $8.24 \mathrm{E}+06$ & 19 & $3.19 \mathrm{E}+06$ & 595 & 242 & 110 & 97.08 & 56.57 & & 5.69 \\
\hline 3 & 1 & 13 & 91 & $1.50 \mathrm{E}+07$ & 34 & $5.60 \mathrm{E}+06$ & 607 & 426 & 145 & 100.7 & 67.59 & 153.99 & 12 \\
\hline 4 & 2 & 6 & 52 & 9.37E+06 & 19 & $42 \mathrm{E}+06$ & 555 & 260 & 118 & 103 & 60.36 & & 7.03 \\
\hline 4 & 1 & 14 & 55 & $1.35 \mathrm{E}+07$ & 20 & $.89 \mathrm{E}+06$ & 409 & 372 & 164 & 103.3 & .39 & 181.77 & \\
\hline 0 & 2 & 42 & 97 & $1.70 \mathrm{E}+07$ & 35 & $.14 \mathrm{E}+06$ & 570 & 466 & 157 & 104.5 & 70.69 & & \\
\hline 4 & 1 & 4 & 139 & $6.17 \mathrm{E}+06$ & 48 & $2.13 E+06$ & 2252 & 162 & 47 & 109 & 78.3 & 154.55 & 8. \\
\hline 17 & 1 & 17 & 61 & $1.64 \mathrm{E}+07$ & 21 & $5.65 E+06$ & 372 & 429 & 185 & 109 & 65.98 & 188.37 & 27.0 \\
\hline 1 & 2 & 33 & 73 & $7.32 \mathrm{E}+06$ & 25 & $2.51 \mathrm{E}+06$ & 997 & 190 & 75 & 109.9 & 69.43 & 180.57 & 5.0 \\
\hline 0 & 1 & 10 & 107 & $1.63 \mathrm{E}+07$ & 35 & $5.33 E+06$ & 657 & 405 & 136 & 114.9 & 78.23 & 173.32 & 2.1 \\
\hline 5 & 2 & 37 & 31 & $5.85 \mathrm{E}+06$ & 10 & $1.89 \mathrm{E}+06$ & 530 & 143 & 88 & 115.9 & 56.21 & 82 & 0.4 \\
\hline 0 & 2 & 2 & 61 & $6.89 \mathrm{E}+06$ & 19 & $2.15 \mathrm{E}+06$ & 885 & 163 & 74 & 120.6 & 71.71 & 3.49 & 1.01 \\
\hline 1 & 2 & 43 & 243 & $1.16 \mathrm{E}+07$ & 72 & $3.43 \mathrm{E}+06$ & 2100 & 260 & 61 & 126.8 & 97.3 & 165.11 & 7.27 \\
\hline 0 & 2 & 12 & 34 & $3.89 \mathrm{E}+06$ & 10 & $1.14 \mathrm{E}+06$ & 875 & 87 & 54 & 126.9 & 2.3 & 287.74 & 3.8 \\
\hline 1 & 1 & 1 & 67 & $1.38 \mathrm{E}+07$ & 19 & $3.93 E+06$ & 484 & 299 & 135 & 132 & 79.11 & 232.36 & 3.5 \\
\hline 3 & 1 & 3 & 32 & $8.84 \mathrm{E}+06$ & 9 & $2.49 \mathrm{E}+06$ & 362 & 189 & 123 & 132.2 & 62.78 & 314.47 & 47.67 \\
\hline 5 & 1 & 5 & 70 & $1.56 \mathrm{E}+07$ & 19 & $4.23 E+06$ & 449 & 322 & 146 & 137.8 & 82.88 & 242 & 34.9 \\
\hline 3 & 2 & 5 & 133 & $1.11 \mathrm{E}+07$ & 36 & $3.01 \mathrm{E}+06$ & 1197 & 228 & 76 & 138.9 & 96.06 & 06.41 & 5.83 \\
\hline 6 & 2 & 38 & 97 & $1.42 \mathrm{E}+07$ & 26 & $3.81 \mathrm{E}+06$ & 682 & 289 & 113 & 140.1 & 90.79 & & $30 .{ }^{2}$ \\
\hline 6 & 1 & 16 & 75 & $1.82 \mathrm{E}+07$ & 20 & $4.85 E+06$ & 412 & 369 & 163 & 0.3 & & & 4.6 \\
\hline 19 & 2 & 1 & 34 & $6.98 \mathrm{E}+06$ & 9 & $1.85 \mathrm{E}+06$ & 487 & 140 & 91 & 0.6 & 67.28 & & .38 \\
\hline 12 & 1 & 12 & 58 & $1.17 \mathrm{E}+07$ & 15 & $3.02 \mathrm{E}$ & 497 & 230 & 117 & 144.3 & 81.74 & 68 & 40.68 \\
\hline 1 & 1 & 11 & 71 & $1.35 \mathrm{E}+07$ & 18 & $3.41 \mathrm{E}+06$ & 528 & 259 & 121 & 147.4 & 87.85 & 262.23 & 38.02 \\
\hline 2 & 2 & 24 & 64 & $1.52 \mathrm{E}+07$ & 16 & $3.80 \mathrm{E}+06$ & 421 & 288 & 142 & 149.6 & 36.52 & 276.72 & 40.7 \\
\hline 26 & 2 & 8 & 72 & $2.27 \mathrm{E}+07$ & 18 & $5.68 \mathrm{E}+06$ & 317 & 431 & 201 & 149.7 & 89.35 & 266.23 & 38.5 \\
\hline 53 & 2 & 35 & 120 & $7.83 \mathrm{E}+06$ & 29 & $1.89 \mathrm{E}+06$ & 1532 & 144 & 53 & 155.2 & 103.6 & 241.12 & 31.69 \\
\hline 9 & 1 & 9 & 123 & $2.81 \mathrm{E}+07$ & 29 & $6.62 \mathrm{E}+06$ & 438 & 504 & 186 & 158.7 & 106 & 246.27 & 32.32 \\
\hline 39 & 2 & 21 & 68 & $2.13 \mathrm{E}+07$ & 16 & $5.00 \mathrm{E}+06$ & 320 & 379 & 187 & 158.8 & 92.28 & 292.71 & 43.01 \\
\hline 48 & 2 & 30 & 86 & $1.65 \mathrm{E}+07$ & 20 & 3.85 & 520 & 292 & 129 & 160.9 & & & 9.1 \\
\hline 40 & 2 & 22 & 36 & $7.42 \mathrm{E}+06$ & 8 & $1.65 \mathrm{E}+06$ & 485 & 125 & 86 & 166.7 & 92 & 3.83 & 61.9 \\
\hline 35 & 2 & 17 & 64 & +07 & 14 & $3.0 \varepsilon$ & 455 & 233 & 12 & 170.4 & & & 48.83 \\
\hline 8 & 1 & 8 & 51 & +07 & 11 & 2.24 & 492 & 170 & 10 & & & & .0 \\
\hline 7 & 2 & 9 & 80 & $1.47 \mathrm{E}+07$ & 17 & $3.12 \mathrm{E}+06$ & 545 & 237 & 11 & 175.7 & 4.5 & & $45.7 \mathrm{~s}$ \\
\hline 3 & 2 & 25 & 76 & $1.55 \mathrm{E}+07$ & 16 & $3.25 \mathrm{E}+06$ & 492 & 247 & 122 & 177.2 & 103.8 & 324.56 & 47.4 \\
\hline 8 & 2 & 10 & 68 & $1.62 \mathrm{E}+07$ & 14 & $3.34 \mathrm{E}+06$ & 419 & 254 & 133 & 180.9 & 102.3 & 347.14 & 51.5 \\
\hline 1 & 2 & 23 & 97 & $1.73 \mathrm{E}+07$ & 19 & $3.39 E+06$ & 561 & 257 & 117 & 190.5 & 117.1 & 328.77 & 46.7 \\
\hline 2 & 2 & 4 & 149 & $1.62 \mathrm{E}+07$ & 29 & $3.15 E+06$ & 920 & 239 & 88 & 192.1 & 129.6 & 295.82 & 38.4 \\
\hline 0 & 2 & 32 & 94 & $2.53 \mathrm{E}+07$ & 18 & $4.84 E+06$ & 372 & 367 & 171 & 194.7 & 118.2 & 341.37 & 48.9 \\
\hline & 2 & 15 & 89 & $1.21 \mathrm{E}+07$ & 17 & $.31 \mathrm{E}+06$ & 735 & 176 & 84 & 195.1 & 116.8 & 348.37 & 50.3 \\
\hline
\end{tabular}




$\begin{array}{cccccccccccccc}47 & 2 & 29 & 67 & 1.44 \mathrm{E}+07 & 10 & 2.15 \mathrm{E}+06 & 465 & 163 & 101 & 247.1 & 129.3 & 534.31 & 80.27 \\ 25 & 2 & 7 & 87 & 1.96 \mathrm{E}+07 & 13 & 2.93 \mathrm{E}+06 & 444 & 222 & 121 & 247.6 & 140.1 & 480.14 & 71.25 \\ 54 & 2 & 36 & 93 & 1.80 \mathrm{E}+07 & 13 & 2.52 \mathrm{E}+06 & 517 & 191 & 104 & 264.3 & 150.1 & 510.78 & 75.72 \\ 21 & 2 & 3 & 99 & 9.71 \mathrm{E}+06 & 12 & 1.18 \mathrm{E}+06 & 1020 & 89 & 51 & 303.6 & 169.9 & 600.64 & 89.48 \\ 6 & 1 & 6 & 35 & 1.01 \mathrm{E}+07 & 4 & 1.15 \mathrm{E}+06 & 347 & 88 & 83 & 313.7 & 117.9 & 1175.57 & 148.95 \\ 59 & 2 & 41 & 42 & 1.12 \mathrm{E}+07 & 4 & 1.06 \mathrm{E}+06 & 376 & 81 & 76 & 375.2 & 143.6 & 1378.86 & 176.33 \\ 44 & 2 & 26 & 86 & 2.82 \mathrm{E}+07 & 8 & 2.62 \mathrm{E}+06 & 305 & 199 & 137 & 390.4 & 195.9 & 912.19 & 136.52 \\ 38 & 2 & 20 & 101 & 1.94 \mathrm{E}+07 & 8 & 1.53 \mathrm{E}+06 & 522 & 116 & 80 & 456 & 230.9 & 1054.42 & 158.36 \\ 36 & 2 & 18 & 103 & 2.23 \mathrm{E}+07 & 8 & 1.74 \mathrm{E}+06 & 461 & 132 & 90 & 464.7 & 235.6 & 1073.15 & 161.25 \\ 45 & 2 & 27 & 80 & 1.28 \mathrm{E}+07 & 6 & 9.63 \mathrm{E}+05 & 623 & 73 & 57 & 477.8 & 219.7 & 1288.88 & 187.77 \\ 52 & 2 & 34 & 191 & 1.67 \mathrm{E}+07 & 14 & 1.23 \mathrm{E}+06 & 1142 & 93 & 49 & 495 & 295.5 & 903.49 & 132.73 \\ 57 & 2 & 39 & 130 & 2.17 \mathrm{E}+07 & 9 & 1.51 \mathrm{E}+06 & 598 & 114 & 74 & 520 & 275.3 & 1127.19 & 170.45 \\ 37 & 2 & 19 & 84 & 2.28 \mathrm{E}+07 & 5 & 1.36 \mathrm{E}+06 & 369 & 103 & 88 & 593.4 & 258.5 & 1760.38 & 249.77 \\ 49 & 2 & 31 & 94 & 1.66 \mathrm{E}+07 & 5 & 8.82 \mathrm{E}+05 & 567 & 67 & 57 & 660.5 & 289.8 & 1936.73 & 277\end{array}$

PARAMETERS:

nM: Number of mounts

rhoD: Effective track density $\left(\mathrm{tr} / \mathrm{cm}^{2}\right)$

$\mathrm{nD}$ : Count for fluence monitor (tr)

uGlass: Uranium concentration of glass standard (ppm)

zeta: zeta factor $\left(\mathrm{a} \mathrm{cm}^{2} / \mathrm{tr}\right)$

zetaSE: standard error of zeta factor $\left(\mathrm{a} \mathrm{cm}^{2} / \mathrm{tr}\right)$

sSC: size of square counter $\left(\mathrm{cm}^{2}\right)$

$\begin{array}{ccccccc}\mathrm{nM} & \text { rhoD } & \mathrm{nD} & \text { uGlass } & \text { zeta } & \text { zetaSE } & \text { SSC } \\ 1 & 5.23 \mathrm{E}+05 & 6523 & 39.8 & 145.39 & 7.04 & 1.00 \mathrm{E}-08 \\ 2 & 5.25 \mathrm{E}+05 & 6537 & 39.8 & 145.39 & 7.04 & 1.00 \mathrm{E}-08\end{array}$


Table S4: Detailed zircon fission-track grain and sample data. 485 